\title{
Charge sharing between pixels in the spectral Medipix2 x-ray detector
}

\author{
H. Zeller ${ }^{1}$, S. Dufreneix ${ }^{1,3}$, M. Clark ${ }^{1}$, \\ P.H. Butler ${ }^{1}$, A.P.H. Butler ${ }^{2}$ \\ ${ }^{1}$ Department of Physics and Astronomy \\ ${ }^{2}$ Department of Electrical and Computer Engineering \\ University of Canterbury \\ Christchurch, New Zealand \\ ${ }^{3}$ Universite Paris-Sud 11 \\ Paris, France
}

\author{
N. Cook ${ }^{4}$, L. Tlustos ${ }^{5}$ \\ ${ }^{4}$ Medical Physics and Bioengineering \\ Christchurch Hospital \\ Christchurch, New Zealand \\ ${ }^{5} \mathrm{CERN}$ \\ Geneva, Switzerland
}

\begin{abstract}
This paper gives an overview of the Medipix 2 x-ray detector and its use in medical imaging, with the MARS-CT scanner (MARS, Medipix All Resolution System) as an example. The Medipix2 chip is a photon counting pixel detector with the ability of energy discrimination. It was developed at CERN and is composed of a sensor layer bump bonded to electronics layer. It has 256x256 pixels, each one covering an area of $55 \times 55 \mu \mathrm{m}^{2}$. Furthermore, every pixel can be read out separately. The MARS-CT scanner uses these properties to scan biological objects obtaining multi-energy (spectral) $x$-ray images with high contrast between materials and high spatial resolution. Charge sharing is the phenomenon by which the electron-hole charge cloud, induced in the sensor layer by an absorbed photon, is detected by a cluster of neighbouring pixels. Each pixel in the cluster generates a signal corresponding to its fraction of the cloud, so the detector will record several photons each of lower energies. This effect has to be considered with the use of Medipix2, because of its small pixels and the hybrid architecture. The effect was measured and a simulation modelled with the aim to reconstruct the spectrum removing the distortion of the detection process.
\end{abstract}

\section{INTRODUCTION}

For the LHC (Large Hadron Collider) experiments at CERN (Conseil Européen pour la Recherche Nucléaire) noise free pixel detectors were developed. The Medipix3 Collaboration is migrating this technology to other imaging applications to profit, among others, from its key advantage the freedom of noise.

The first Medipix chip was developed at the beginning of the 1990s with the ability of single photon counting. The pixel size was $170 \times 170 \mu \mathrm{m}^{2}$. This is quite high, limiting its spatial resolution. With the development of Medipix2 the area covered by one pixel was shrunk to $55 \times 55 \mu^{2}$. More importantly the ability to discriminate between energies was included. Furthermore, Medipix2 is designed as a hybrid detector - the electronic layer is separated from the detector layer, allowing the use of different materials for the latter.

The MARS-CT team in Christchurch is developing a spectral CT scanner with this chip family as detecting device. In spectral CT a broad energy spectrum is measured, providing more information per image taken, due to detecting not only the intensity of an x-ray beam but also its spectral distribution. The evaluation of scanned mice and atherosclerotic plaque shows promising results in spatial resolution and material contrast [1].

The small pixel size and the hybrid architecture are both advantages, but are the source of a detrimental effect called charge sharing. Charge sharing is the effect of recording more photons each of lower energy rather than the real number of incident photons each of higher energy. Thus, there is a distortion of the measured spectrum. This is a consequence of charge diffusion perpendicular to the applied electric field in the detecting layer. It causes artefacts in the acquired image to occur and the theoretical spatial and energy resolution is not achieved.

\section{MEDIPIX2 PIXEL DETECTOR}

The Medipix2 pixel detector is divided into two layers (see figure 1). For the detecting layer, different semiconducting materials like silicon ( $\mathrm{Si}$ ), gallium arsenide (GaAs), or cadmium telluride (CdTe) can be used. This layer is bump bonded to a $256 \times 256(64 \mathrm{k})$ pixel matrix on a CMOS chip. This chip is capable of measuring negative as well as positive 
charges, providing together with the hybrid architecture a very flexible usage.

Furthermore the chip has a large sensitive area $(87 \%$ of the entire chip area is detecting area) and other chips can be mounted on 3 sides without having dead areas in between. This is possible because the electronics are concentrated on one side of the chip, therefore arrays can be built forming bigger sensitive regions. Quad detectors (an array of 4 Medipix2 chips bonded to a larger sensor chip) have been trialled [2].

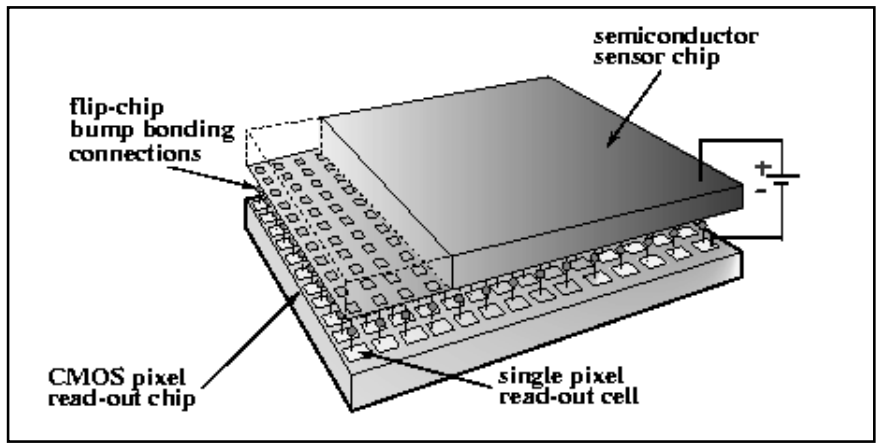

Figure 1. Medipix2 pixel detector

Each pixel is configured over 8 bits. Six of them define a lower and upper threshold, one serves as a mask for the chip in case of malfunction or excessive noise and the last one for input charge testing.

Every photon interacting with the sensor layer creates electron-hole pairs. Depending on a bias voltage, the electrons or holes are collected by the $64 \mathrm{k}$ electrodes. The signal measured by each pixel's electronics is compared to the two thresholds; if it is between them then a 13 bit digital counter is incremented. This is Medipix2's photocounting mode where it has the ability to discriminate between the incoming photon energies.

The matrix to be read out has 256 columns of 256 pixels each of 13 bits. There are two possible ways to perform the readout, one is in serial mode, with a readout time of less than $9 \mathrm{~ms}$, and the other in parallel mode within $266 \mu \mathrm{s}$.

The Medipix3 generation of this chip is under development at CERN providing new features, among others, a simultaneous measurement of photons in 8 different energy bins and a charge sharing correction.

\section{MARS-CT SCANNER}

The MARS-CT team in Christchurch is developing a spectral CT scanner using the Medipix detector family. In the Siemens and GE dual-energy CT scanners two energies are used and therefore two different materials can be readily distinguished. In multi-energy spectroscopic scans, on the other hand, more information is provided by the transmission of a broad spectrum beam through a biological object if it is detected and processed. Hence more information about the sample is acquired in less time.

Due to the dependence of attenuation coefficient from photon energy and illuminated matter, and by using the energy discrimination features of the Medipix2 chip, images containing additional information to each other are obtained. Therefore a higher contrast between different materials has been achieved in comparison to the two clinical dual energy scanners. Furthermore the small pixel size provides images with high spatial resolution. Measurements confirming this advantages where made, among others, on mice and atherosclerotic plaque [3].

By rotating the scanner around the object of interest a 3D image can be reconstructed out of the $2 \mathrm{D}$ projections. The $3 \mathrm{D}$ reconstruction naturally profits from the capabilities of the Medipix 2 chip.

The next step is the replacement of Medipix2 with Medipix3. Due to the new feature of simultaneously measuring 8 energy bins, there will be a decrease in scanning time and a reduction in the doses to which the scanned object is exposed but, at the same time, increasing the amount of information obtained. Such improvements in the detectors will get us closer to in vivo scans.

\section{Charge Sharing IN MediPIX2 PiXel Detector}

Due to the combination of small pixel size and hybrid architecture of the Medipix2 detector, an effect called charge sharing is observed.

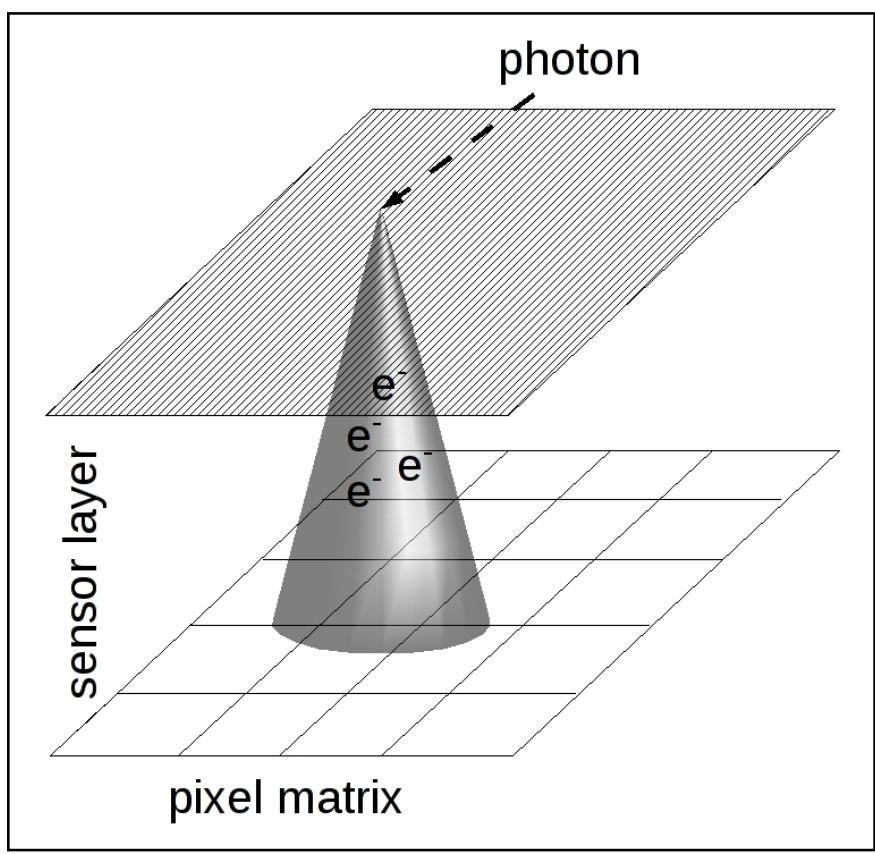

Figure 2. Charge sharing effect

When a photon with energy in the $\mathrm{keV}$ region is absorbed by the sensor layer an electron-hole cloud is produced. The energy gap between valence and conduction band is in the eV region, so the number of excited electrons is of order $10^{3}$, assuming that the photon has not been scattered before. The charges are accelerated in direction of the electrodes due to the electric field in between them. Because of diffusion effects (charge concentration gradients in the sensor layer) the cloud has the tendency to broaden perpendicular to the electric field causing a cone shaped charge distribution. Therefore an incoming photon with certain energy can be detected by more 


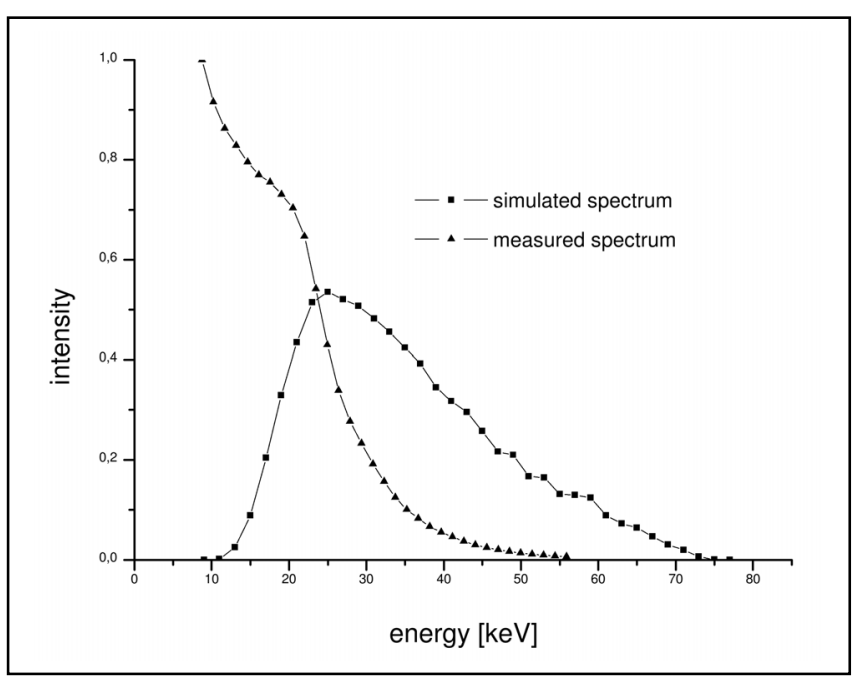

Figure 3. Comparision between the simulated energy spectrum without charge sharing effect and the measured one

than one pixel (see figure 2). This results in a signal of more photons with lower incident energies, and correspondingly fewer counts in the high energy bins. This is a source for image artefacts, a decrease in both spatial and energy resolution, and the need of longer exposure times, because material characteristics in the spectrum are smeared out [4].

\section{A. Comparison between expected and distorted spectrum}

In this study the broad energy spectrum of an x-ray tube was measured with a Medipix2 detector. The shape of the graph does not agree with the theoretical expectation, which were obtained through Monte Carlo simulation (see comparison of both spectra in figure 3). For the measurement, a Perspex cylinder with $2 \mathrm{~cm}$ radius was illuminated by a rectangular $\mathrm{x}$-ray beam of $10 \mathrm{x} 7 \mathrm{~mm}^{2}$ cross section. The beam was generated by an $\mathrm{x}$-ray tube with $75 \mathrm{kVp}$ at $0.150 \mathrm{~mA}$ and a tungsten anode, and detected by a Medipix 2 chip with silicon as sensor material. The simulated distribution was obtained by simulating the set up using BEAMnrc (CNational Research Council of Canada), a simulation software based on EGS4 Monte Carlo code. It can be seen that for x-rays within the energy range of $10-100 \mathrm{keV}$, which is used in CT scanners, the amount of charge sharing is significant. The area under both graphs was normalized to the same value. This does not take into account that the detected graph artificially measures more photons that actually are absorbed by the sensor layer. Therefore the difference between both spectra should be even bigger. These data agree with comparison of measurement and simulation made by H.-E. Nillson et al. [5].

\section{B. Modelling of charge sharing effect}

To model the charge sharing effect a code was written which calculates the amount of charge every pixel detects. The approach taken was to divide a cluster of 9 pixels, 1 central and 8 neighbouring, into 1 central pixel and one "superpixel" surrounding the first one. It was assumed that the electron and energy distribution is homogeneous over the whole circular area overlapping the square pixels. Further assumptions were that the photon is absorbed without having been scattered, and fluorescence effects of atoms can be neglected. Finally for the simulation, only one radius was used. Thus we did not take

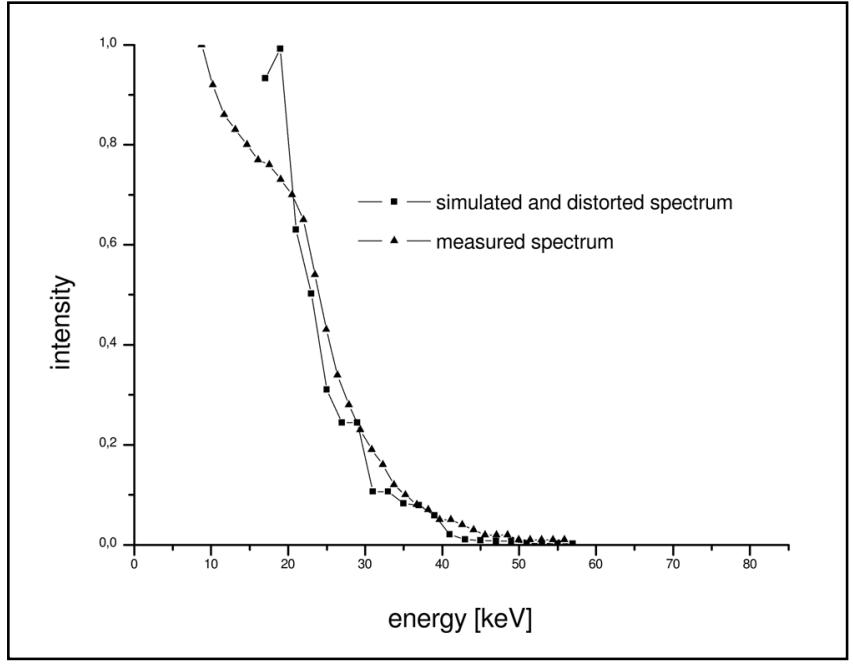

Figure 4. Simulated spectrum is distorted due to charge sharing and sensor layer efficiency

into account different absorption depths due the different energies of the incident photons.

With this method we calculated the probability of a photon, with certain incident energy $E_{\text {in }}$, being measured in a specific energy bin $\left(E_{\text {det }}\right)$ of the detector. The resulting probabilities are listed in table I.

TABLE I. CORRECTION COEFFICIENTS FOR CHARGE SHARING

\begin{tabular}{|c|c|c|c|c|c|c|c|c|}
\hline$f_{i j}$ & $\begin{array}{c}16.2- \\
19.7\end{array}$ & 23.3 & 26.8 & 30.3 & 33.9 & 37.5 & 41 & $>41$ \\
\hline$<16.2$ & 0.692 & 0.617 & 0.550 & 0.501 & 0.456 & 0.423 & 0.393 & 0.314 \\
\hline 19.7 & 0.226 & 0.130 & 0.106 & 0.100 & 0.089 & 0.077 & 0.071 & 0.058 \\
\hline 23.3 & & 0.195 & 0.122 & 0.097 & 0.089 & 0.083 & 0.073 & 0.057 \\
\hline 26.8 & & & 0.177 & 0.108 & 0.089 & 0.082 & 0.076 & 0.057 \\
\hline 30.3 & & & & 0.160 & 0.104 & 0.082 & 0.075 & 0.057 \\
\hline 33.9 & & & & & 0.146 & 0.095 & 0.077 & 0.057 \\
\hline 37.4 & & & & & & 0.135 & 0.094 & 0.058 \\
\hline 41 & & & & & & & 0.122 & 0.062 \\
\hline$>41$ & & & & & & & & 0.280 \\
\hline
\end{tabular}

Energies in the first row $\left(\mathrm{E}_{\mathrm{det}}\right)$ and column $\left(\mathrm{E}_{\text {in }}\right)$ are in $\mathrm{keV}$.

\section{Compensation for charge sharing}

The best result in image reconstruction is obtained with a spectrum only affected by the object and not by the detector. Therefore changes in the energy distribution due to effects in the detector should be corrected. For charge sharing there is a technique called "spectrum stripping", which uses monochromatic sources with a known spectrum to compare to the response of the detector. From there the corrections are calculated for a broad spectrum [6]. Our approach was to model the effect as described in section IV.B. and use the coefficients in table I to take charge sharing into account.

$$
I_{\text {det }}(i)=\Sigma_{j}\left(a_{j} \cdot f_{i j} \cdot I_{i n}(j)\right)
$$


Figure 4 shows the simulated spectrum of figure 3 distorted due to charge sharing. This was done by using the coefficients from table $I$ in equation (1) $\left(I_{\text {det }}\right.$ and $I_{\text {in }}$ are the intensity of the distorted and not distorted spectrum respectively). Furthermore, it was taken into account that the efficiency for absorbing photons in the silicon layer decreases with increasing energy [7]. Simulating a monochromatic photon beam and a silicon slab of $300 \mu \mathrm{m}$ thickness with BEAMnrc, gives absorption probabilities $\mathrm{a}_{\mathrm{j}}\left(\mathrm{E}_{\mathrm{in}}\right)$ for the sensor layer used on the Medipix2 chip. Table II shows the energy dependent values, which were used for figure 4 . Both graphs agree well over $20 \mathrm{keV}$. Below $20 \mathrm{keV}$ the method predicts a higher fluency than measured. This can be traced back to the assumptions made to model the charge sharing effect. At lower energies the photon is absorbed closer to the surface of the sensor and results in a bigger cone radius. Furthermore, fewer electrons are excited in this case. For these reasons, the assumption of a uniform electron distribution, does not hold. We leave it to future work to include this in calculations of the charge distribution.

\section{TABLE II. CORRECTION COEFFICIENTS FOR SENSOR LAYER EFFICIENCY}

\begin{tabular}{|c|c|}
\hline Energy $[\mathbf{k e V}]$ & $\mathbf{a}_{\mathbf{j}}\left(\mathbf{E}_{\mathbf{i n}}\right)$ \\
\hline 15 & 0.492 \\
\hline 18 & 0.321 \\
\hline 20 & 0.244 \\
\hline 22 & 0.188 \\
\hline 25 & 0.130 \\
\hline 30 & 0.074 \\
\hline 40 & 0.029 \\
\hline 50 & 0.012 \\
\hline
\end{tabular}

\section{CONCLUSION}

The Medipix2 chip is a powerful detector which offers through its architecture a high flexibility. The images taken with MARS-CT scanner present a high spatial resolution and material contrast but there are still artefacts due to different effects that if excluded would improve the data quality.
It was shown that the charge sharing effect is significant in the energy scale of interest and a proposal was made for a first correction. The next step is to reconstruct the spectrum of every pixel and analyse the resulting image. Further work will be put into improving the underlying model.

\section{ACKNOWLEDGMENT}

The authors would like to thank all members of the Medipix2 collaboration, the Medipix3 Collaboration, and the MARS CT team for their great support.

\section{REFERENCES}

[1] R. Zainon, N. de Ruiter, P. H. Butler, A. P. H. Butler, S. P. Gieseg, N. J. Cook, M. A. Hurrell, "Spectroscopic x-ray computed tomography imaging of plaque and arteries using the Medipix detector" presented at The New Zealand Institute of Physics Conference 2009.

[2] X. Llopart, M. Campbell, R. Dinapoli, D. San Segundo, E. Pernigotti, "Medipix2, a $64 \mathrm{k}$ pixel readout chip with $55 \mu \mathrm{m}$ square elements working in single photon counting mode" Nuclear Science Symposium Conference Records, 2001 IEEE Volume 3, 4-10 Nov. 2001, pp. 1484 1488.

[3] M. Firsching, A. P. Butler, N. Scott, N. Anderson, T. Michel, G. Anton, "Contrast agent recognition in small animal CT using the Medipix2 detector", Nuclear Instruments and Methods in Physics Research A 607 (2009), pp. 179-182.

[4] M. Maiorino, G. Pellegrini, G. Blanchot, M. Chmeissani, J. Garcia, R. Martinez, M. Lozano, C. Puigdengoles, M. Ullan, "Charge-sharing observations with CdTe pixel detector irradiated with a ${ }^{57} \mathrm{Co}$ source" Nuclear Instruments and Methods in Physics Research A 563 (2006), pp. 177-181.

[5] H.-E. Nilsson, C.G. Mattsson, B. Norlin, C. Frojdh, K. Bethke, R. De Vries, "Evaluation of the charge-sharing effects on spot intensity in XRD setup using photon-counting pixel detectors" Nuclear Instruments and Methods in Physics Research A 563 (2006), pp. 182-186.

[6] M. Firsching, P.T. Talla, T. Michel, G. Anton, "Material resolving Xray imaging using spectrum reconstruction with Medipix2" Nuclear Instruments and Methods in Physics Research A 591 (2008), pp. 19-23.

[7] D.W. Davidson, J. Watt, L. Tlustos, B. Mikulec, M. Campbell, K. Mathieson, V. O'Shea, K.M. Smith, M. Rahman, "Detective Quantum Efficiency of the Medipix Pixel Detector", IEEE Transactions on Nuclear Science, vol. 50, No. 5, October 2003, pp. 1659-1663 